\title{
Polyester composite reinforced with fiber of Mauritia flexuosa treated with alkali: Impact energy, chemical composition and surface topography
}

\author{
Mackinley Epiquien Saavedra1, Katherine Lavado Lázaro ${ }^{1}$, Meliza Lindsay Rojas, Dr. ${ }^{2}$, Leslie Lescano, MSc. ${ }^{3}$, Jesús \\ Sánchez-González, MSc. ${ }^{3}$,Guillermo Linares, Dr. ${ }^{3}$, Danny Chávez Novoa, MSc', Alexander Vega Anticona, Dr ${ }^{4}$, *. \\ ${ }^{1}$ Escuela de Ingeniería de Materiales, Universidad Nacional de Trujillo,Perú,mepiquien@unitru.edu.pe, \\ klavadol@unitru.edu.pe \\ 2Dirección de Investigación y Desarrollo,Universidad Privada del Norte,Perú,meliza.rojas@upn.edu.pe \\ ${ }_{3}^{3}$ Departamento de Ciencias Agroindustriales, Universidad Nacional de Trujillo,Perú, llescano@unitru.edu.pe, \\ jsanchezg@unitru.edu.pe,glinares@unitru.edu.pe. \\ ${ }^{4}$ Departamento de Ingeniería de Materiales, Universidad Nacional de Trujillo,Perú, dchavez@unitru.edu.pe, \\ *corresponding author: \\ avega@unitru.edu.pe \\ Av. Juan Pablo s/n, Phone 044633952
}

\begin{abstract}
This work evaluated the influence of the concentration of $\mathrm{NaOH}(100,200$ and $300 \mathrm{~g} / \mathrm{L} \mathrm{NaOH})$ in the mercerization process at $25^{\circ} \mathrm{C}$ for $1 \mathrm{~h}$, and of the percentage $(15,20$ and $25 \% \mathrm{~m} / \mathrm{m})$ of Mauritia flexuosa fibers on impact resistance in a polyester matrix composite. The polyester matrix composite was obtained by the compression molding process, at a constant pressure of $24 \mathrm{MPa}$. The obtained plates had a dimension of $130 \mathrm{~mm}$ long $x 80 \mathrm{~mm}$ wide $x 3.4$ mm thick. Specimens for the impact assay were extracted from the obtained plates under the EN ISO 179-1 standard. Likewise, the fiber-reinforced composites without treatment were also evaluated, all the fibers were characterized before and after the $\mathrm{NaOH}$ treatment by means of the chemical characterization of the fiber, absorption percentage, scanning electron microscopy (SEM) and Fourier transform infrared spectroscopy (FT-IR). The results showed that with a higher concentration of $\mathrm{NaOH}$, a decrease in extractives, lignin and hemicellulose was obtained, while the cellulose content increased. Additionally, it was shown that the higher the $\mathrm{NaOH}$ concentration, the hydrophilic character of the fiber decreases. On the other hand, by means of FT-IR, it was identified that the functional groups related to the fiber composition vary before and after mercerization. While the scanning electron microscopy (SEM) showed morphological changes of the fiber where impurities, waxes and fats were observed for the untreated fiber and a rougher surface and free of impurities for the treated fibers.
\end{abstract}

The highest impact resistance value was obtained by $25 \%$ fiber reinforced composites without treatment with an average value of $39.62 \mathrm{~kJ} / \mathrm{m} 2$, a value that represents an increase of $298.34 \%$ with respect to the impact resistance of the polyester matrix. without reinforcement. The results concluded that the weight percentage of fiber increases impact resistance while chemical treatment increases fiber-matrix adhesion and influences the failure mechanism of polyester matrix compounds, however, decreases impact resistance. Thus, the compounds reinforced with $25 \%$ fiber and treated with 100, 200 and $300 \mathrm{~g} / \mathrm{L}$ of $\mathrm{NaOH}$ reported average values of impact resistance of $23.02 \mathrm{~kJ} / \mathrm{m} 2,28.01 \mathrm{~kJ} / \mathrm{m} 2$ and 20.48 $\mathrm{kJ} / \mathrm{m} 2$ respectively.

Keywords - fiber Mauritia flexuosa mercerization polyester matrix reinforced composites.

Digital Object Identifier (DOI):

http://dx.doi.org/10.18687/LACCEI2020.1.1.516

ISBN: 978-958-52071-4-1 ISSN: 2414-6390

$1^{\text {th }}$ LACCEI International Multi-Conference for Engineering, Education, and Technology: "Engineering, Integration, and Alliances for a Sustainable Development" "Hemispheric Cooperation for Competitiveness and Prosperity on a Knowledge-Based Economy", July 27-31, 2020, Virtual Edition. 


\title{
Compuestos de poliéster reforzados con fibra de Mauritia flexuosa tratada con álcali: Energía de impacto, composición química y topografía superficial.
}

\author{
Mackinley Epiquien Saavedra ${ }^{1}$, Katherine Lavado Lázaro ${ }^{1}$, Meliza Lindsay Rojas, Dr. ${ }^{2}$, Leslie Lescano, MSc. ${ }^{3}$, Jesús \\ Sánchez-González, MSc. ${ }^{3}$,Guillermo Linares, Dr. ${ }^{3}$, Danny Chávez Novoa, MSc ${ }^{4}$, Alexander Vega Anticona, Dr ${ }^{4}{ }^{*}$. \\ ${ }^{1}$ Escuela de Ingeniería de Materiales, Universidad Nacional de Trujillo, Perú, mepiquien@unitru.edu.pe, \\ klavadol@unitru.edu.pe \\ ${ }^{2}$ Dirección de Investigación y Desarrollo, Universidad Privada del Norte, Perú, meliza.rojas@upn.edu.pe \\ ${ }^{3}$ Departamento de Ciencias Agroindustriales, Universidad Nacional de Trujillo, Perú, 1 lescano@unitru.edu.pe, \\ jsanchezg@unitru.edu.pe,glinares@unitru.edu.pe. \\ ${ }^{4}$ Departamento de Ingeniería de Materiales, Universidad Nacional de Trujillo,Perú, dchavez@unitru.edu.pe,
}

*corresponding author:

avega@unitru.edu.pe

Av. Juan Pablo s/n, Phone 044633952

Resumen - El presente trabajo evaluó la influencia de la concentración de $\mathrm{NaOH}(100,200$ y $300 \mathrm{~g} / \mathrm{L}$ de $\mathrm{NaOH})$ del proceso de mercerización a $25{ }^{\circ} \mathrm{C}$ por $1 \mathrm{~h}$, y del porcentaje en peso $(15,20$ y $25 \%$ ) de fibras de Mauritia flexuosa sobre la resistencia al impacto en un compuesto de matriz poliéster. Los compuestos de matriz poliéster fueron obtenidos por el proceso de moldeo por compresión, a una presión constante de 24 MPa. Las placas obtenidas tuvieron una dimensión de $130 \mathrm{~mm}$ de largo $x 80 \mathrm{~mm}$ de ancho $x 3.4 \mathrm{~mm}$ de espesor. De las placas obtenidas se extrajeron probetas para el ensayo de impacto bajo la norma EN ISO 179-1. Así mismo fueron evaluados los compuestos reforzados con fibra sin tratamiento, todas las fibras fueron caracterizadas antes $y$ después del tratamiento mediante la caracterización química de la fibra, porcentaje de absorción, microscopia electrónica de barrido (SEM) y espectroscopia infrarroja por transformada de Fourier (FT-IR), a fin de determinar de forma indirecta la modificación de los compuestos químicos de las fibras vegetales. Los resultados mostraron que a mayor concentración de $\mathrm{NaOH}$ se obtuvo una disminución de extractivos, lignina y hemicelulosa (estos dos últimos de mayor interés desde el punto de vista de propiedades mecánicas de las fibras) mientras que el contenido de celulosa aumentó. Adicionalmente, se evidenció que a mayor concentración de $\mathrm{NaOH}$ el carácter hidrofílico de la fibra disminuye. Por otro lado, mediante FT-IR, se identificó que los grupos funcionales relacionados a la composición de la fibra varían antes y después de la mercerización. Mientras que la microscopia electrónica de barrido (SEM) mostró cambios morfológicos de la fibra en donde se observó impurezas, ceras y grasas para la fibra sin tratamiento y una superficie más rugosa y libre de impurezas para las fibras tratadas. El mayor valor de resistencia al impacto fue el obtenido por los compuestos reforzados con $25 \%$ de fibra sin tratamiento con un valor promedio de $39.62 \mathrm{~kJ} / \mathrm{m}^{2}$, valor que representa un aumento del $298.34 \%$ con respecto a la resistencia al impacto de la matriz poliéster sin refuerzo. Los resultados concluyeron que el porcentaje en peso de fibra incrementa la resistencia al impacto mientras que el tratamiento químico incrementa la adhesión de fibra-matriz e influye en el mecanismo de falla de los compuestos de matriz poliéster, sin embargo, disminuye la resistencia al impacto. Siendo así que los compuestos reforzados con $25 \%$ de fibra y tratados con 100, 200 y $300 \mathrm{~g} / \mathrm{L}$ de $\mathrm{NaOH}$ reportaron valores promedios de resistencia al impacto de $23.02 \mathrm{~kJ} / \mathrm{m}^{2}, 28.01$ $\mathrm{kJ} / \mathrm{m}^{2}$ y $20.48 \mathrm{~kJ} / \mathrm{m}^{2}$ respectivamente. La evaluación medio ambiental rebasa los límites de la presente investigación.

Palabras clave: fibra, Mauritia flexuosa, mercerización, matríz poliéster, compuestos reforzados

\section{INTRODUCCIÓN}

Actualmente la preocupación ambiental debido al elevado uso de materiales poliméricos sintéticos ha generado un interés creciente en el uso de fibras naturales como materiales de refuerzo para compuestos poliméricos alternativos. Las fibras naturales son los componentes más importantes de diversas aplicaciones industriales, como los textiles, la fabricación de papel, el embalaje y los materiales de construcción. En las múltiples industrias, las fibras que se emplearon en primer lugar fueron las que la propia naturaleza ofrecía. Sin embargo, aunque existen más de 500 fibras naturales, muy pocas son las que pueden utilizarse industrialmente, pues no todas las materias se pueden hilar, ni todas las fibras orgánicas son aprovechables para convertirlos en tejidos. Dado que las fibras naturales son materiales renovables y biodegradables, los productos fabricados con dichos materiales también son amigables con el medio ambiente [1].

Las fibras naturales tienen muchas ventajas deseables sobre las fibras sintéticas, por ejemplo buenas propiedades acústica, aislamiento térmico, baja densidad, bajo costo y alta resistencia a la flexión, además de ser no toxicas y

Digital Object Identifier (DOI):

http://dx.doi.org/10.18687/LACCEI2020.1.1.516

ISBN: 978-958-52071-4-1 ISSN: 2414-6390

18th LACCEI International Multi-Conference for Engineering, Education, and Technology: "Engineering, Integration, and Alliances for a Sustainable Development" "Hemispheric Cooperation for Competitiveness and Prosperity on a Knowledge-Based Economy", July 27-31, 2020, Virtual Edition. 
ambientalmente amigables [2]. Las fibras naturales están compuestas de celulosa, hemicelulosa, lignina, pectinas, ceras y sustancias solubles en agua. Sin embargo, el inconveniente más serio de estas fibras naturales es su naturaleza hidrofílica causando una débil unión interfacial entre la fibra y la matriz de los compuestos de matriz plástica. Además, diversas impurezas físicas y la presencia de grupos hidroxilo en la superficie de la fibra crean dificultades para ser utilizados como materiales de refuerzo. Sin embargo, el rendimiento mecánico de un material compuesto depende de múltiples factores, entre ellos, la orientación, tamaño, procedencia, morfología y la naturaleza propia de las fibras, sin dejar de lado las de la matriz, además la forma de unión entre las fibras y la matriz también juega un papel muy importante. [3]

La palma de aguaje (Mauritia flexuosa), presenta una amplia distribución desde la cuenca amazónica hasta la isla de Trinidad (Trinidad y Tobago), llegando a ocupar más de 5 mil hectáreas de la amazonia peruana. Es por ello que es uno de los productos más aprovechados por el poblador, el fruto como fuente de alimento, las fibras para la fabricación textil, las hojas para interiores de vivienda y la madera como fuente de energía. Sin embargo, esta materia prima en materiales de ingeniería no se ha explorado adecuadamente ya que dichas fibras poseen propiedades atractivas para su uso como refuerzo en materiales compuestos [4]. En este contexto, pocos estudios se han venido realizando en cuanto a caracterización y el uso de fibras de Mauritia flexuosa. Por ejemplo, da Silva Santos, et al. [5] demostraron que los materiales compuestos (prensado a 6.0 Ton por $1.5 \mathrm{~h}$ a 150 ${ }^{\circ} \mathrm{C}$ ) a base resina de cardanol-formaldehído reforzados con fibra Mauritia flexuosa tratadas con $\mathrm{NaOH}$ mostraron los mejores resultados de adherencia entre fibra y matriz mediante microscopia electrónica por barrido (SEM). La microscopia mostró que el proceso de mercerización indujo rugosidad en la superficie de la fibra, esto es debido a la eliminación de compuestos de baja masa molar, dejando cavidades en la superficie. Mientras [6] estudiaron la composición química (cantidad de lignina y extractivos), contenido de humedad, espectroscopia infrarroja por transformada de Fourier FTIR, por TGA se determinó alto contenido de humedad y estabilidad térmica que se alcanza alrededor de los $200^{\circ} \mathrm{C}$, difracción de rayos $\mathrm{X}$ (estructura de celulosa I con un índice de cristalinidad de $44.6 \%$ y $51.9 \%$,), ensayos mecánicos y estudios morfológicos.

Por otro lado, Algunos estudios han evaluado la aplicación de tratamientos en fibras para que, mediante modificaciones en la estructura y composición, se mejore su aplicación y propiedades. En este sentido, se han aplicado tratamientos con álcali en diferentes concentraciones (mayormente 5-15\%) en fibra de agave (Mylsamy and Rajendran [7], fibra de la fruta Borassus [8] [9], fibra de Fique [10], fibra de Saharan aloe vera [11], fibra de Abaca [12], fibra de la hoja de palma de Areca [13], tratamientos con peróxido de benzoilo, álcali, permanganato de potasio y ácido esteárico en fibra de Sansevieria cilíndrica [14]. En general, en los estudios previamente mencionados, utilizando ensayos mecánicos y técnicas como microscopía electrónica de barrido (SEM), caracterización térmica por TGA, FTIR, entre otros, se demostró que las fibras tratadas desempeñan un papel importante en el aumento de la resistencia a la flexión y la resistencia al impacto de los materiales compuestos elaborados con las fibras naturales. Además, fue demostrado que con los tratamientos ocurre la eliminación de hemicelulosas, lignina y otras impurezas superficiales de la superficie de la fibra, lo que promovió mejores propiedades mecánicas. Por otro lado, Athijayamani, et al. [15] demostraron que las propiedades mecánicas tales como resistencia a la tracción, flexión e impacto del compuesto hibrido pueden variar en función de la humedad. Al respecto, los tratamiento realizados en fibras disminuyen la tendencia de absorción de agua [14] [12], obteniendo mejores propiedades.

En este trabajo de investigación se pretende mejorar la resistencia al impacto del compuesto de matriz polimérica de poliéster, para lo cual se propone el empleo de la fibra de aguaje (Mauritia flexuosa) con tratamiento superficial y la incorporación en porcentaje en peso, como refuerzo, para producir un material compuesto tenaz, resistente y de bajo peso.

\section{MATERIALES Y MÉTODOS}

\section{A. Diseño de Investigación.}

Se aplicó el diseño experimental tipo bifactorial con tres niveles. Las variables en estudio y sus niveles se muestran en la Tabla 1, resultando un total de 9 tratamientos con 3 repeticiones cada uno. Adicionalmente se realizaron ensayos Control en un total de 3 probetas para resina poliéster y 3 probetas reforzadas con Mauritia Flexuosa al 15\%, 20\% y $25 \%$ de fibra sin tratamiento, todo ello hace un total de 39 ensayos de impacto.

TABLA 1. NIVELES DE VARIABLES DE ESTUDIO.

\begin{tabular}{|ll|l|}
\hline \multicolumn{2}{|c|}{ VARIABLES INDEPENDIENTE } & NIVELES DE ESTUDIO \\
\hline A. & Concentración de $\mathrm{NaOH}$ & $100,200,300 \mathrm{~g} / \mathrm{L}$ \\
\hline B. & Porcentaje en peso de fibra & $15 \%, 20 \%, 25 \%$ \\
\hline
\end{tabular}

\section{B. Procedimiento Experimental.}

a) Proceso de obtención de fibra de cogollo de aguaje (Mauritia flexuosa)

Los cogollos de aguaje fueron recolectados en la provincia de Moyobamba, departamento de San Martín, Perú. Utilizando navajas, los cogollos fueron cortados en láminas longitudinalmente de manera que se mantenga la integridad 
de las fibras. Posteriormente las fibras extraídas se dejaron secar a temperatura de ambiente por tres dias.

Una vez seco, se cortaron las fibras en tamaños de 8-8.5 $\mathrm{cm}$ y se la separó en fracciones de $160 \mathrm{~g}$. Posteriormente se realizaron dos etapas de limpieza. Para la primera limpieza, se sumergió la fibra en agua destilada por un tiempo de 15 min a una temperatura de $60^{\circ} \mathrm{C}$. Para la segunda limpieza, se preparó una solución alcohólica 1:1 (agua:alcohol). La solución se calentó hasta alcanzar una temperatura entre 60 a $80{ }^{\circ} \mathrm{C}$, posteriormente se sumergió la fibra y se mantuvo durante 30 minutos, manteniendo la temperatura en el rango mencionado.

\section{b) Mercerización de fibras}

Para la mercerización se preparó las soluciones de hidróxido de sodio y agua destilada (100, 200 y 300 g/L). Una vez preparadas las soluciones, se añadió las fibras en las soluciones de hidróxido de sodio respectivas, por un tiempo de 1 hora a temperatura de ambiente. Posteriormente, para remover el exceso de hidróxido de sodio, las fibras fueron sumergidas en una solución de 5\% V/V de ácido acético para neutralizar así la fibra. Finalmente se lavó la fibra con agua destilada y se secaron en una estufa a $60{ }^{\circ} \mathrm{C}$ durante 8 horas.

\section{c) Microscopia Electrónica de Barrido (SEM).}

Las fibras deshidratadas fueron previamente recubiertas con una fina capa de oro por deposición de plasma. Luego, la morfología de las fibras se observó en un microscopio electrónico de barrido (FEI, Quanta 650, USA) operado a un voltaje de aceleración de $30 \mathrm{kV}$ con magnificaciones de $200 \mathrm{X}$ a $1600 X$.

\section{d) Caracterización química de la fibra}

Las fibras tratadas y no tratadas (mercerización) fueron evaluadas en función de su composición química, y capacidad de absorción de agua. En este sentido la secuencia fue:

\section{d.1 Determinación de Humedad.}

La humedad de la fibra se determinó según el "Standard Method for Determination of Total Solids in Biomass" de Ehrman [16]. Para ello las muestras (2g.) fueron deshidratadas a $105^{\circ} \mathrm{C}$, utilizando una estufa (MEMMERT, Alemania) hasta peso constante.

\section{d.2 Determinación de Extractivos.}

Para este procedimiento se utilizó el "Standard Method for the Determination of Extractives in Biomass" de Tina Ehrman (1994). Primero se pesó 2 gramos de cada muestra (Sin tratar, 100, 200 y 300g/L de $\mathrm{NaOH}$ ), se armaron pequeños paquetes de fibras, utilizando papel filtro. Luego se utilizó el equipo Soxhlet utilizando como solvente de extracción etanol al 96\%. Durante el proceso se realizó el control contante de la temperatura, la velocidad de reflujo y la velocidad de calentamiento para obtener de cuatro a cinco intercambios de solvente (sifonada) por hora. Este procedimiento se realizó por 24 horas. Posterior a ello el solvente se eliminó, para finalmente secar las fibras en estufa $\left(105^{\circ} \mathrm{C}\right)$ y desecador, hasta peso constante.

\section{d.3 Determinación de Holocelulosa.}

Se pesó $1 \mathrm{~g}$ de la muestra seca libre de extractos, se agregó $80 \mathrm{~mL}$ de agua caliente, $0.5 \mathrm{~mL}$ de ácido acético glacial $\left(\mathrm{CH}_{3} \mathrm{COOH}\right)$ y se agitó. Después se añadió $1 \mathrm{~g}$ de clorito de sodio $(\mathrm{NaClO} 2)$, se realizó una agitación lenta, se tapó y se colocó a baño maría hasta $70{ }^{\circ} \mathrm{C}$. Después de $1 \mathrm{~h}$, se agregó $0.5 \mathrm{~mL}$ de ácido acético y $1 \mathrm{~g}$ de clorito de sodio, se agitó nuevamente. Estas proporciones se agregaron $\mathrm{x} 3$ veces hasta que la muestra tomó un color blanquecino. La muestra se dejó reaccionar durante 24 horas. Al concluir el tiempo, se filtró la muestra y se lavó con alcohol de 96\%. Luego se dejó secar a $45^{\circ} \mathrm{C}$.

\section{d.4 Determinación de Celulosa.}

Para este procedimiento se utilizó el Standard Method for Determination of Alpha-, beta- and gamma-cellulose in pulp (Reaffirmation of T $203 \mathrm{~cm}-99$ ). Para ello se pesó $0.3 \mathrm{~g}$ de muestra blanqueada (obtenida en el proceso anterior), se agregó $15 \mathrm{~mL}$ de $\mathrm{NaOH}$ al $17.5 \%$, se dispersó completamente la pulpa usando un dispensador de pulpa, luego se agregó 5 $\mathrm{mL}$ de $\mathrm{NaOH}$ al $17.5 \%$, se agitó y se colocó en baño maría a $25{ }^{\circ} \mathrm{C}$. Después de $30 \mathrm{~min}$, se añadió $20 \mathrm{~mL}$ de agua destilada, se agitó y se dejó reposar por $30 \mathrm{~min}$. Finalmente, se agitó la suspensión y se filtró, los primeros $4 \mathrm{~mL}$ de filtrado fueron descartados conservando el resto del filtrado.

\section{d.5 Alfa Celulosa}

Se utilizó $0.25 \mathrm{~mL}$ del filtrado de pulpa (obtenido anteriormente), se agregó $1 \mathrm{~mL}$ de $\mathrm{K}_{2} \mathrm{Cr}_{2} \mathrm{O}_{7}$ a $0.5 \mathrm{~N}$, se agregó $5 \mathrm{~mL}$ de $\mathrm{H}_{2} \mathrm{SO}_{4}$ cc agitando lentamente. Se dejó reposar durante $15 \mathrm{~min}$, luego se agregó $2.25 \mathrm{~mL}$ de agua destilada y se dejó enfriar a temperatura ambiente. Posteriormente, se agregó 4 gotas de ferroína (indicador) y se tituló con ácido sulfúrico ferrosos $0.1 \mathrm{~N}$ (previamente valorado) hasta conseguir un viraje de color verdoso a rojizo, obteniendo el volumen de gasto.

Para los cálculos se realizó una valoración en blanco, sustituyendo el filtrado de pulpa con $1.25 \mathrm{~mL}$ de $\mathrm{NaOH}$ y $1.25 \mathrm{~mL}$ de agua destilada.

\section{d.6 Beta y Gamma Celulosa (Hemicelulosa)}

Se midió el filtrado de pulpa en una probeta de $50 \mathrm{~mL}$, se adicionó $9 \mathrm{~mL}$ de ácido sulfúrico $\mathrm{H}_{2} \mathrm{SO}_{4} 3 \mathrm{~N}$, se mezcló, se colocó en baño maría $\left(70-90^{\circ} \mathrm{C}\right)$ hasta coagular, luego se dejó reposar y se centrifugó por un tiempo de 10 minutos a $40 \mathrm{rpm}$. Posteriormente se tomó $0.5 \mathrm{~mL}$ del sobrenadante y se agregó 
$1 \mathrm{~mL}$ de $\mathrm{K}_{2} \mathrm{Cr}_{2} \mathrm{O}_{7}$ al $0.5 \mathrm{~N}$ y $9 \mathrm{~mL}$ de ácido sulfúrico concentrado. Luego se dejó reposar durante $15 \mathrm{~min}$, se agregó $2.25 \mathrm{~mL}$ de agua destilada y se dejó enfriar a temperatura ambiente. Posteriormente, se agregó 4 gotas de ferroína (indicador) y se tituló con ácido sulfúrico ferrosos $0.1 \mathrm{~N}$ (previamente valorado) hasta conseguir un viraje de color verdoso a rojizo, obteniendo el volumen de gasto.

Para los cálculos se realizó una prueba en blanco sustituyendo la solución con $1.25 \mathrm{~mL}$ de hidróxido de sodio $(\mathrm{NaOH}) 17.5 \%, 1.25 \mathrm{~mL}$ de agua destilada y $2.5 \mathrm{~mL}$ de ácido sulfúrico $3 \mathrm{~N}$.

\section{d.7 Determinación de Lignina.}

Para este procedimiento se utilizó el Standard Method for Determination of Acid-Insoluble Lignin in Biomass de David Templeton and Ehrman [17].

\section{d.8 Lignina Klason (Ácido sulfúrico al 72\%)}

Se pesó $5 \mathrm{~g}$ de muestra sin extractivos y se colocaron en tubos de vidrio. Luego, se agregó $\mathrm{H}_{2} \mathrm{SO}_{4}$ al $72 \%$ (por cada $100 \mathrm{mg}$ se adicionó $1 \mathrm{~mL}$ de ácido sulfúrico). Después los tubos se llevaron a baño maría a temperatura $30^{\circ} \mathrm{C}$ agitando frecuentemente hasta su total solución. Después de una hora, se diluyó la muestra $84 \mathrm{~mL}$ de agua destilada. Luego paso a la autoclave a $120^{\circ} \mathrm{C}$ por 60 minutos. La dispersión se filtró (al vacío), este filtrado se usó en lignina soluble y el residuo sólido se lavó con agua caliente para remover todo el ácido, el residuo fue utilizado en Lignina insoluble.

\section{d.9 Lignina Soluble}

Se preparó la muestra denominada blanco, colocando en un tubo con tapa $10 \mathrm{~mL}$ de ácido sulfúrico al $72 \%$ y $10 \mathrm{~mL}$ de agua destilada agitando la solución. La muestra se llevó a evaluación con el espectrofotómetro.

\section{d.10 Lignina Insoluble}

El residuo sólido mencionado en esta sección, se secó a $105^{\circ} \mathrm{C}$ por 2 horas, se llevó a peso constante. Posteriormente, se calcinó la muestra en una mufla a $575^{\circ} \mathrm{C}$, hasta obtener peso constante.

\section{d.11 Determinación del \% de absorción de agua.}

Para el ensayo de absorción de lagua de las fibras se realizó bajo la norma ASTM D570. Para esto se tomó la masa inicial de las fibras sin y con tratamiento (mi), luego se sumergió en agua y se anotó las masas ( $\mathrm{mf}$ ) en intervalos de tiempo (1, 2, 3, 4, 5,6 y 24 horas), el ensayo se realizó a temperatura ambiente. El porcentaje de absorción de agua se calculó mediante la siguiente formula.

$\%$ Absorcion de agua $=\frac{m_{f}-m_{i}}{m_{i}} \times 100$ e) Espectroscopia de trasmisión infrarroja por transformada de Fourier (FTIR)

Para el ensayo de espectroscopia de trasmisión infrarroja por transformada de Fourier (FTIR) se llevó acabo utilizando un espectrofotómetro modelo IR Spectrum Two (PerkinElmer, USA). Luego de una limpieza y calibración previa, el ensayo de FTIR se realizó a 0.5 g de Fibra de Mauritia Flexuosa sin tratamiento, y con tratamiento de 100 $\mathrm{g} / \mathrm{L}$ y $300 \mathrm{~g} / \mathrm{L}$ de $\mathrm{NaOH}$ colocadas sobre la punta de diamante la cual es ajustada con el tornillo de ajuste. Luego se procedió a realizar el barrido correspondiente, el software del equipo realizó la gráfica (\% Transmitancia vs número de onda $\left(\mathrm{cm}^{-}\right.$ $1))$.

\section{f) Conformado de placas del compuesto de resina poliéster} y fibras de Mauritia flexuosa

Para determinar las proporciones de mezcla para el curado de la resina poliéster, se realizó la preparación a diferentes dosificaciones de solvente, acelerante y endurecedor, típicos de esta resina, teniendo como criterio de elección, la energía de impacto (Charpy). Así se determinó que por cada kg de resina poliéster Anypol 100, se utilizarían $350 \mathrm{~g}$ de monómero de $10 \mathrm{~g}$ de octoato de cobalto al 6\%estireno y 25 cc de peróxido de M.E.K.

Una vez definidas la dosificación de la resina poliéster, se preparó un molde de acero, con medidas de $130 \mathrm{~mm}$ (largo) x $80 \mathrm{~mm}$ (ancho) x 3.4 mm (espesor), en el cual se realizó el conformado de placas de resina reforzada con fibra Mauritia Flexuosa $(15 \%, 20 \%$ y $25 \%$ peso) sin tratamiento y tratadas previamente con 100, 200 y $300 \mathrm{~g} / \mathrm{L}$ de $\mathrm{NaOH}$. Las masas utilizadas se calcularon considerando el volumen del molde y densidades de los materiales, luego se verifico por masas de matriz y fibras los porcentajes peso utilizados, existiendo una variación entre estos datos menores al $4.2 \%$

Después de habilitado el molde (limpio y encerado), se colocó una pequeña cantidad de la mezcla de resina en el molde para luego colocar la fibra Mauritia Flexuosa de manera ordenada, se vertió la resina sobrante y por último se tapó el molde de manera cuidadosa para no desordenar la fibra.

\section{g) Moldeo por compresión.}

Para el moldeo por compresión se utilizó un equipo de compresión (TecnoTest, Italia). La presión utilizada fue de 24 $\mathrm{MPa}$. El molde se mantuvo en el equipo por 12 horas, pasado ese tiempo se procedió a retirar la placa del molde y se deja curar por 72 horas más. Todo el proceso se realizó a temperatura ambiente

h) Preparación de probetas para ensayo de impacto según norma EN ISO 179-01. 
Se procedió a cortar las placas (elaboradas en el proceso de compresión) para obtener las probetas de impacto (Figura 1) con dimensiones según la norma EN ISO 179-1. En el caso de solo resina poliéster se consideró la norma para plásticos no reforzados, mientras que las probetas conteniendo la fibra fueron elaboradas de acuerdo con la norma para plásticos reforzados (Tabla 2).

TABLA 2: DIMENSIONES DE PROBETAS PARA ENSAYO TIPO CHARPY A PLÁSTICOS REFORZADOS Y NO REFORZADOS (Norma ISO 179 - 01)[18].

\begin{tabular}{|l|l|l|l|l|}
\hline Muestra & $\begin{array}{l}\text { Largo } \\
(\mathbf{m m}) \\
\mathbf{l}\end{array}$ & $\begin{array}{l}\text { Ancho } \\
(\mathbf{m m}) \\
\mathbf{b}\end{array}$ & $\begin{array}{l}\text { Espesor (mm) } \\
\text { h }\end{array}$ & $\begin{array}{l}\text { Span } \\
(\mathbf{m m}) \\
\text { L }\end{array}$ \\
\hline $\begin{array}{l}\text { Plásticos } \\
\text { no } \\
\text { reforzados }\end{array}$ & $80 \pm 0.20$ & $10 \pm 0.20$ & $4 \pm 0.20$ & $62 \pm 0.20$ \\
\hline $\begin{array}{l}\text { Plásticos } \\
\text { reforzados }\end{array}$ & $25 \mathrm{~h}$ & 10 o 15 & 3 & $20 \mathrm{~h}$ \\
\hline
\end{tabular}

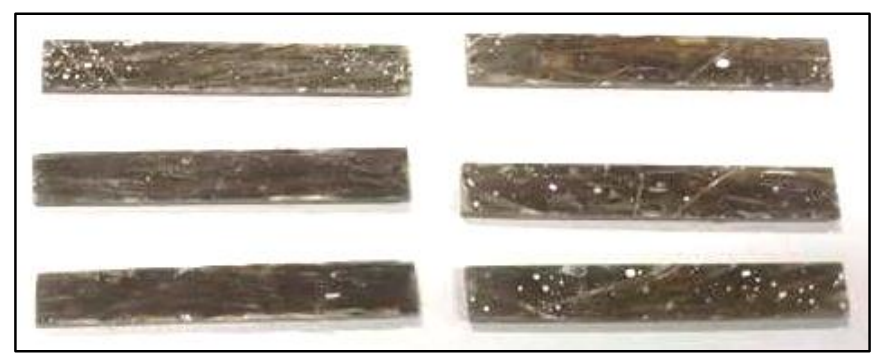

Fig. 1. Probetas de impacto obtenidas según la norma EN ISO 179-01 para plásticos reforzados.

\section{i) Ensayo de impacto Charpy}

Las probetas ya preparadas se ensayaron en una máquina de ensayo tipo Charpy (TERCO, Suecia.) con una carga máxima de 15 Joules $(1 \mathrm{~J}=1 \mathrm{Nm})$ y escala de $0.1 \mathrm{~J}$. Las probetas fueron puestas en la porta probetas para luego ser
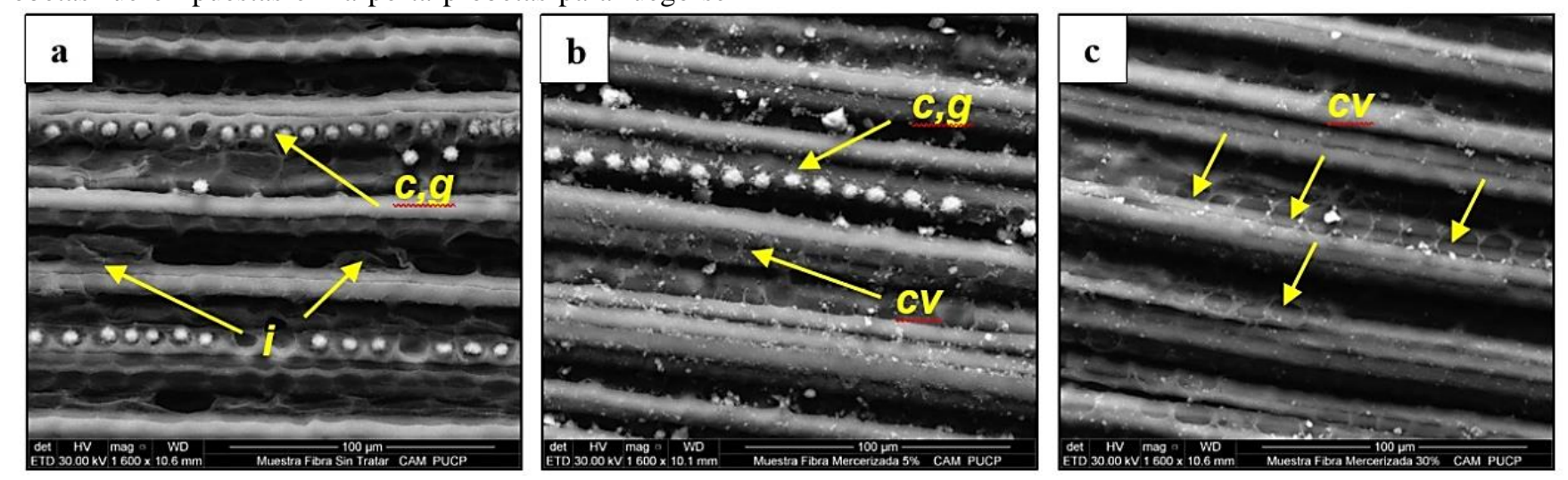

Fig. 2: Imagen SEM de fibra Mauritia Flexuosa, magnificación de 1600X. a) Sin tratamiento; b) tratada con $100 \mathrm{~g} / \mathrm{L}$ de NaOH; c) tratada con 300 g/L de NaOH.

Las fibras tratadas mostraron superficies de mayor rugosidad, incremento de cavidades ( $c v)$ y menor cantidad de impurezas. Estos cambios sugieren ser la consecuencia del tratamiento impactadas por el péndulo de impacto. Los datos fueron registrados. La resistencia al impacto Charpy, $a_{c N}$, (2) en $\left(\mathrm{kJ} / \mathrm{m}^{2}\right)$, se define como la energía de impacto en la rotura respecto al área de la sección transversal inicial de la superficie impactada. Dónde: h: espesor de la probeta $(\mathrm{mm})$; b: ancho de la probeta $(\mathrm{mm}) ; \mathrm{E}_{\mathrm{c}}$ : energía absorbida en la rotura $(\mathrm{J})[19]$.

$$
a_{c N}=\frac{E_{B}}{h b} \times 10^{3}
$$

\section{j) Análisis estadístico}

Se realizó el análisis de varianza y prueba " $F$ " y se determinó la influencia de cada una de las variables en estudio a un diseño experimental bifactorial. El análisis de varianza (ANAVA) se efectuó a un nivel de confianza del $95 \%$.

\section{RESULTADOS Y DISCUSION}

\section{A. Microscopia electrónica de barrido (SEM)}

La Figura 2 muestras imágenes SEM en donde se indica los cambios morfológicos que presenta la fibra Maurita flexuosa de las fibras sin tratamiento (a), y tratadas a una concentración de 100 (b) y 300 (c) g/L de $\mathrm{NaOH}$. La fibra sin tratamiento mostró una superficie lisa con presencia de impurezas $(i)$, ceras y grasas $(c, g)$ (ver detalles en flechas). Según Sinha, et al. [12] informa que debido a los residuos cerosos, la humectabilidad superficial de las fibras vegetales, en matriz polimérica, se reduce, dando por resultado la disminución de adherencia entre ellos, además presentar baja rugosidad superficial. Por otro lado, en las fibras tratadas con $\mathrm{NaOH}$ se observan cambios en la superficie de las mismas, respecto a las no tratadas. 
proporcionan un mayor número de puntos de anclaje generando así una mayor adherencia a la matriz polimérica. Por lo tanto, la modificación en la topografía superficial de la fibra mediante los tratamientos con $\mathrm{NaOH}$ puede influir en las propiedades mecánicas tanto de la fibra, como en la del compuesto que los utiliza como refuerzo.

\section{B. .Caracterización química de fibra Mauritia flexuosa}

TABLA 3. DATOS SOBRE LA CARACTERIZACIÓN QUÍMICA DE LA FIBRA Mauritia flexuosa.

\begin{tabular}{|c|c|c|c|c|c|}
\hline Muestras & $\begin{array}{c}\text { Humedad } \\
(\mathbf{\%})\end{array}$ & $\begin{array}{c}\text { Extractivo } \\
(\boldsymbol{\%})\end{array}$ & $\begin{array}{c}\text { Lignina } \\
(\mathbf{\%})\end{array}$ & $\begin{array}{c}\text { Celulosa } \\
(\mathbf{\%})\end{array}$ & $\begin{array}{c}\text { Hemicelulosa } \\
(\boldsymbol{\%})\end{array}$ \\
\hline ST & $10.44 \%$ & $7.29 \%$ & $29.14 \%$ & $38.81 \%$ & 24.09 \\
\hline Mz -100 & $8.71 \%$ & $4.13 \%$ & $27.43 \%$ & $49.40 \%$ & 17.91 \\
\hline Mz -200 & $8.50 \%$ & $4.11 \%$ & $27.06 \%$ & $49.51 \%$ & 18.24 \\
\hline Mz -300 & $8.19 \%$ & $4.03 \%$ & $26.82 \%$ & $50.48 \%$ & 17.59 \\
\hline
\end{tabular}

La Tabla 3, muestra el contenido de los porcentajes de celulosa, hemicelulosa, lignina, extractivos y humedad de las fibras sin tratamiento (ST), y tratadas a una concentraciones de 100 (Mz-100), 200 (Mz-200) y 300 (Mz-300) g/L de $\mathrm{NaOH}$. Se observó que, al incrmentar la concentración de $\mathrm{NaOH}$, el contenido de humedad, extractivo, lignina y hemicelulosa se reducen, mientras el contenido de celulosa muestra una tendencia creciente de $38.82 \%$ a $50.48 \%$; ello debido a la disminución de lignina y hemicelulosa, lo que ocasiona cambios topográficos en la superficie de la fibra, como fue evidenciado en las imágenes SEM (ver Fig. 2). Según Júnior, et al. [6] ha reportado valores similares de lignina, extractivos y contenido de humedad. Balaji and Nagarajan [11] concluye que el contenido de lignina de la fibra no varía significativamente después del tratamiento alcalino de las fibras, mientras que el contenido de $\alpha$-celulosa aumentó hasta un $7.2 \%$ este aumento puede proporcionar mejores propiedades mecánicas a la fibra, ya que forma una estructura microcristalina con regiones cristalinas de alto orden y regiones amorfas de bajo orden. En el presente estudio, las modificaciones químicas disminuyeron el contenido de hemicelulosa. Por lo que, al disminuir la hemicelulosa, la cual funciona como anclaje entre la lignina (matriz) y celulosa (refuerzo) [14], la celulosa quedo expuesta aumentando su presencia a cuantificar (Tabla 3). Por otro lado, la disminución del contenido de humedad de las fibras tratadas con $\mathrm{NaOH}$ podría indicar que su carácter hidrofílico disminuyo, por lo que el porcentaje de absorción de agua fue evaluado.

\section{Porcentaje de absorción de agua}

La Figura 3 muestra la capacidad de absorción de agua de las fibras Mauritia Flexuosa con y sin tratamiento. La fibra sin tratamiento mostró mayor \% de absorción de agua, respecto a la fibra tratada lo que confirma la disminución del carácter hidrofílico de la fibra, el menor porcentajes de absorción de agua se muestra para las fibras tratadas a una concentración de 300 g/L de $\mathrm{NaOH}$. También se observa que en todas las fibras con y sin tratamiento el porcentaje de absorción es mayor durante las primeras horas, luego esta capacidad, disminuye; según Mwaikambo and Ansell [20] mencionan que existe un grado de saturación de la fibra, es decir que luego de cierto tiempo la fibra ya no podrá seguir absorbiendo agua lo que se llama punto de saturación, el cual según la Figura 3, se daría aproximadamente a las 6 h.

\section{Espectroscopia infrarroja con transformada de Fourier (FT-IR)}

En la Figura 4, se muestra el espectro FT-IR de las fibras sin tratamiento y con tratamiento de mercerizado a concentraciones de 100 y $300 \mathrm{~g} / \mathrm{L}$ de $\mathrm{NaOH}$. En el espectro de fibra sin tratamiento, la $\alpha$-celulosa se encuentra representada un número de onda de $3336 \mathrm{~cm}^{-1}$ siendo asociada a la vibración de estiramiento de los grupos hidroxilos enlazados con hidrogeno $(\mathrm{OH})$. Por lo tanto, en muestras no tratadas, una vibración de estiramiento baja indica un menor porcentaje de celulosa. Por otro lado, los picos $2916 \mathrm{~cm}^{-1}$ y $2848 \mathrm{~cm}^{-1}$ se asocian a la vibración de estiramiento del grupo funcional de los alcanos $(\mathrm{C}-\mathrm{H})$ que representan las moléculas de $\beta$-celulosa y $\gamma$-celulosa respectivamente. El número de onda de $1733 \mathrm{~cm}^{-1}$ representa a la hemicelulosa siendo asocian a la vibración de estiramiento de los grupos funcionales de los carbonilos $(\mathrm{C}=\mathrm{O})$. El pico que se encuentra en un número de onda de $1605 \mathrm{~cm}^{-1}$ representa la presencia de absorción de agua confirmando la naturaleza hidrofílica de la fibra, esta naturaleza hidrofílica de la fibra se puede comprobar con el ensayo de absorción de agua que se realizó a las fibras con y sin tratamiento (ver Figura 3).

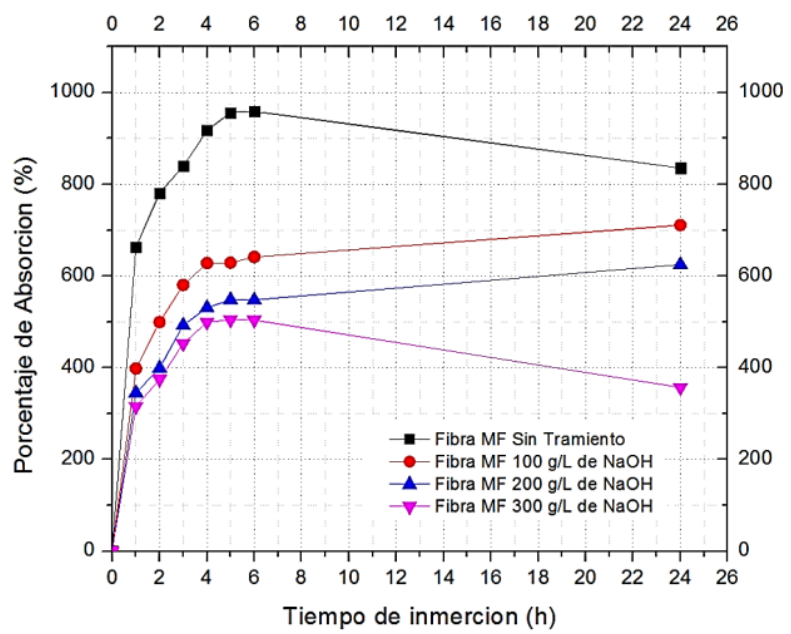

Fig.3. Porcentaje de absorción de agua de la fibra Mauritia Flexuosa con y sin tratamiento.

El pico en el número de onda de $1462 \mathrm{~cm}^{-1}$ asociada a la vibración de estiramiento del grupo funcional de aromáticos representa la molécula de lignina, estos números de ondas tienen relación con los números de onda representados por 
Smith [21]. Según Balaji and Nagarajan [11], la presencia de celulosa, hemicelulosa y lignina en su análisis FT-IR en un rango de 4000 a $500 \mathrm{~cm}^{-1}$, encontrados en la fibra de Saharan sin tratamiento en una número de onda de $3340 \mathrm{~cm}^{-1}, 1750$ $\mathrm{cm}^{-1}, 1525 \mathrm{~cm}^{-1}$ respectivamente, también se reporta la presencia de absorción de agua en un pico de $1600 \mathrm{~cm}^{-1}$ números de onda. Así los picos con números de onda correspondientes a la presencia de hemicelulosa y lignina al presentar una vibración de estiramiento alta indican un mayor porcentaje de estas moléculas en la fibra, guardando esto relación con la caracterización química (Ver Tabla 3).

En cuanto al espectro FT-IR de las fibras tratadas a una concentración de 100 y $300 \mathrm{~g} / \mathrm{L}$ de $\mathrm{NaOH}$, se puede observar que la intensidad de los picos correspondientes a hemicelulosas $\left(1733 \mathrm{~cm}^{-1}\right)$ y lignina $\left(1462 \mathrm{~cm}^{-1}\right)$ disminuye, mientras que el pico correspondiente a glucosa $\left(3336 \mathrm{~cm}^{-1}\right)$ aumentó. Resultados similares fueron reportados por Balaji and Nagarajan [11] después del tratamiento con álcali. Mientras que el pico encontrado con un numero de onda de $1605 \mathrm{~cm}^{-1}$ en la fibra Mauritia Flexuosa que representa la presencia de absorción de agua, dicho pico se encuentra en las fibras tratadas con una menor intensidad ya que disminuye el carácter hidrofílico de la fibra.

\section{E. Resistencia al impacto}

Los resultados de resistencia al impacto promedio obtenidos de los compuestos de matriz poliéster reforzado con fibra a diferentes porcentajes en peso de fibra $(15,20 \mathrm{y}$ $25 \%)$ y concentraciones de $\mathrm{NaOH}(100,200$ y $300 \mathrm{~g} / \mathrm{L})$, ademas de los compuestos reforzados con fibra sin tratamiento alcalino a los diferentes porcentajes en peso de fibra, se muestran en la Tabla 4.

En la Figura 5, se observa el efecto del incremento del porcentaje de la fibra con y sin tratamiento con $\mathrm{NaOH}$, sobre la energía de absorción de impacto (Charpy), de los materiales compuestos de matriz poliéster, los valores de promedio muestran la relación directa entre el porcentaje en peso de fibra (con y sin tratamiento) y la energía de impacto, obteniendo los mayores valores a $25 \%$ en peso de fibras, en todos los casos; los valores de energía de impacto que se consiguieron al incorporar la fibra sin tratamiento en la matriz poliéster, fueron de $23.14 \mathrm{~kJ} / \mathrm{m}^{2}, 27.00 \mathrm{~kJ} / \mathrm{m}^{2}$ y $39.62 \mathrm{~kJ} / \mathrm{m}^{2}$ para los porcentaje en peso de fibra de $15 \%, 20 \%$ y $25 \%$ respectivamente, esto representa un aumento del $174.25 \%$, $203.31 \%$ y $298.34 \%$ con respecto a la energía de impacto promedio de la resina poliéster sin refuerzo (línea discontinua, ver Fig. 5); siendo este valor promedio de 13.28 $\mathrm{KJ} / \mathrm{m}^{2}$, para la dosificación de mezcla mencionado. Según Candido, et al. [22], Yang, et al. [23], el incremento del porcentaje en peso de fibra, aumenta la resistencia al impacto debido a que la fibra absorbe la energía del impacto, interfiriendo en la propagación de grietas de la matriz; durante el impacto, se promueve el desprendimiento de fibra y la propagación de las grietas a lo largo de la interfaz fibra- matriz, ello estaría asociado al proceso de falla por delaminación que presenta la fibra sin tratamiento (Ver Fig. 6). Según Martínez, et al. [24] cuando existe daño interlaminar en los compuestos la resistencia al impacto es mayor.

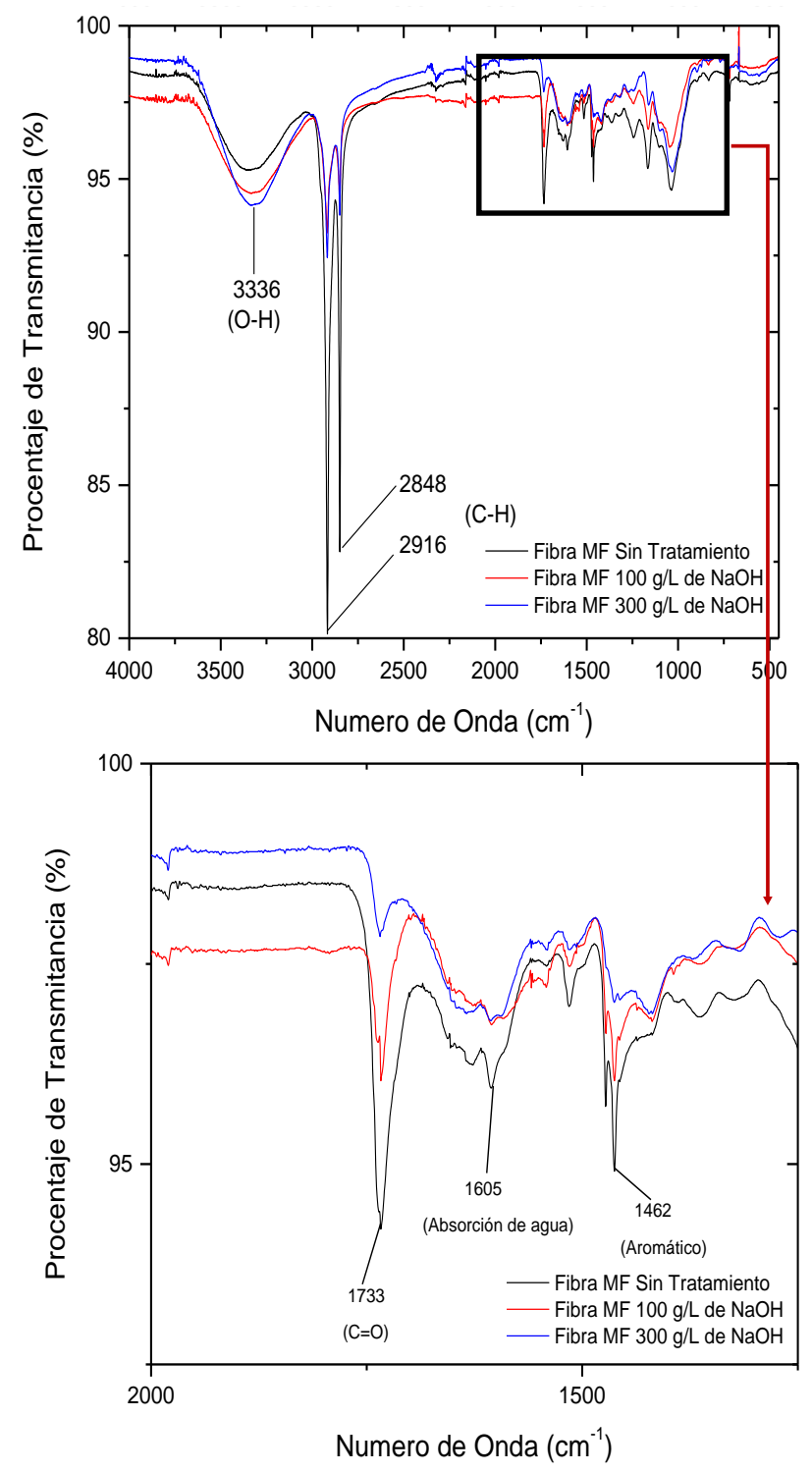

Fig. 4. Espectros FT-IR de fibra sin tratamiento y la fibra tratada a una concentración de 100 y $300 \mathrm{~g} / \mathrm{L}$ de $\mathrm{NaOH}$.

TABLA 4. DATOS PROMEDIOS DE RESISTENCIA AL IMPACTO DE LA RESINA POLIÉSTER, LOS COMPUESTOS SIN MODIFICACIÓN QUÍMICA Y DE LOS COMPUESTOS CON MODIFICACIÓN QUÍMICA SEGÚN LA NORMA EN ISO 179-1.

\begin{tabular}{|l|c|}
\hline MUESTRA & $\begin{array}{l}\text { Resistencia al impacto } \\
\text { promedio }\left(\mathbf{K J} / \mathbf{m}^{2}\right)\end{array}$ \\
\hline ST 15\% & $23.14 \pm 4.42$ \\
\hline ST 20\% & $27.00 \pm 0.71$ \\
\hline ST 25\% & $39.62 \pm 0.15$ \\
\hline
\end{tabular}




\begin{tabular}{|l|l|}
\hline F $15 \%-\mathrm{MZ} 100 \mathrm{~g} / \mathrm{L}$ de $\mathrm{NaOH}$ & $14.51 \pm 1.68$ \\
\hline F $20 \%-\mathrm{MZ} 100 \mathrm{~g} / \mathrm{L} \mathrm{de} \mathrm{NaOH}$ & $19.48 \pm 2.04$ \\
\hline F $25 \%-\mathrm{MZ} 100 \mathrm{~g} / \mathrm{L} \mathrm{de} \mathrm{NaOH}$ & $23.02 \pm 1.67$ \\
\hline F $15 \%-\mathrm{MZ} 200 \mathrm{~g} / \mathrm{L} \mathrm{de} \mathrm{NaOH}$ & $19.95 \pm 1.70$ \\
\hline F $20 \%-\mathrm{MZ} 200 \mathrm{~g} / \mathrm{L} \mathrm{de} \mathrm{NaOH}$ & $25.97 \pm 0.16$ \\
\hline F $25 \%-\mathrm{MZ} 200 \mathrm{~g} / \mathrm{L} \mathrm{de} \mathrm{NaOH}$ & $28.01 \pm 1.04$ \\
\hline F $15 \%-\mathrm{MZ} 300 \mathrm{~g} / \mathrm{L} \mathrm{de} \mathrm{NaOH}$ & $13.38 \pm 1.95$ \\
\hline F $20 \%-\mathrm{MZ} 300 \mathrm{~g} / \mathrm{L} \mathrm{de} \mathrm{NaOH}$ & $17.50 \pm 1.73$ \\
\hline F $25 \%-\mathrm{MZ} 300 \mathrm{~g} / \mathrm{L} \mathrm{de} \mathrm{NaOH}$ & $20.48 \pm 1.81$ \\
\hline
\end{tabular}

ST: sin tratamiento; MZ: mercerizado; F: \%fibra.

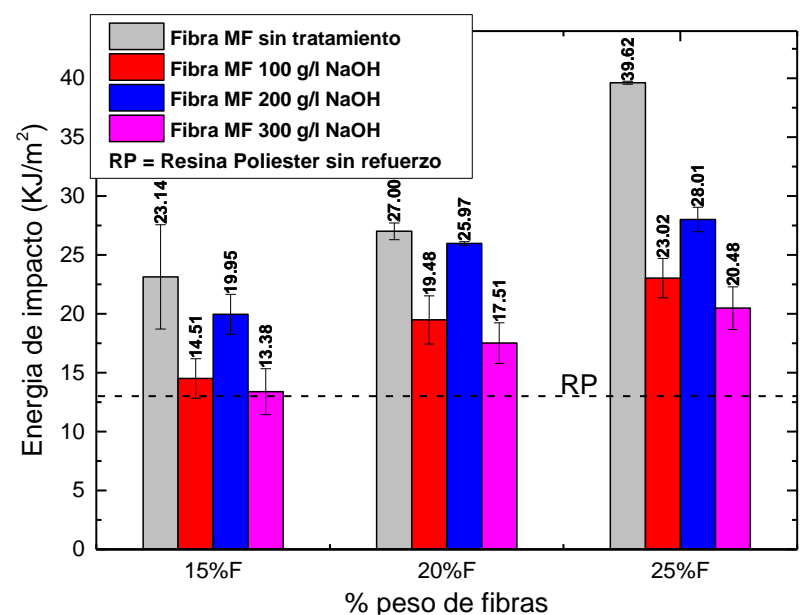

Fig.5. Resistencia al Impacto Vs \% Peso de fibra Mauritia Flexuosa para las fibras sin tratamiento y con tratamiento de $\mathrm{NaOH}(100,200$ y 300 $g / L)$

Por el contrario, en los compuestos de resina poliéster con fibra tratadas con $\mathrm{NaOH}$, la energía al impacto es menor que en los casos similares con fibras sin tratamiento. Sin embargo, comparando entre los datos de los compuestos con fibras tratadas, se aprecia incremento de la energía de impacto, conforme la concentración de $\mathrm{NaOH}$ pasa de 100 $\mathrm{g} / \mathrm{L}$ a $200 \mathrm{~g} / \mathrm{L}$. En la Figura 6 se indica los tipos de daños tanto a la fibra como a la matriz, según Martínez, et al. [24], los mecanismos de daño se dan, en la matriz, de tipo interlaminar (interface) y en las fibras. En la misma figura, se puede apreciar que el compuesto reforzado con $25 \%$ en peso de fibra tratada con $200 \mathrm{~g} / \mathrm{L}$ de $\mathrm{NaOH}$ presenta una mayor delaminación comparado con los tratamientos a (100 y 300) g/L de NaOH. Según Rodríguez, et al. [25], la disminución de la resistencia al impacto promedio de los compuestos reforzados con fibra tratada puede deberse a una disminución de la elasticidad típica necesaria en las fibras vegetales, pues ellas cumplen su función de carga y de transporte, esta disminución, generaría incremento de rigidez con pérdida de elasticidad y por ende menor capacidad de deformación ante el impacto (Ver Fig. 6). Por otro lado, Li, et al. [26], afirma que si la concentración de álcali es elevada, el resultado en fibras, puede ser el debilitamiento o daño a las fibras y consecuentemente una disminución en las propiedades de las mismas, ya que los tratamientos con $\mathrm{NaOH}$ o mercerización son tratamientos de degradación controlada de fibras, estos es generan la modificación de la composición química inicial de fibra (Ver Tabla 3). Para el caso de $300 \mathrm{~g} / \mathrm{L}$ de $\mathrm{NaOH}$, que se utilizó, si bien el grado de adherencia se evidencia mayor (Ver Fig. 6), el modo de daño no permitió delaminación, lo que se tradujo en los bajos valores de absorción de energía de impacto repostados en nuestro caso; según Mallick [27] a altos niveles de adhesión, el modo de falla es quebradizo

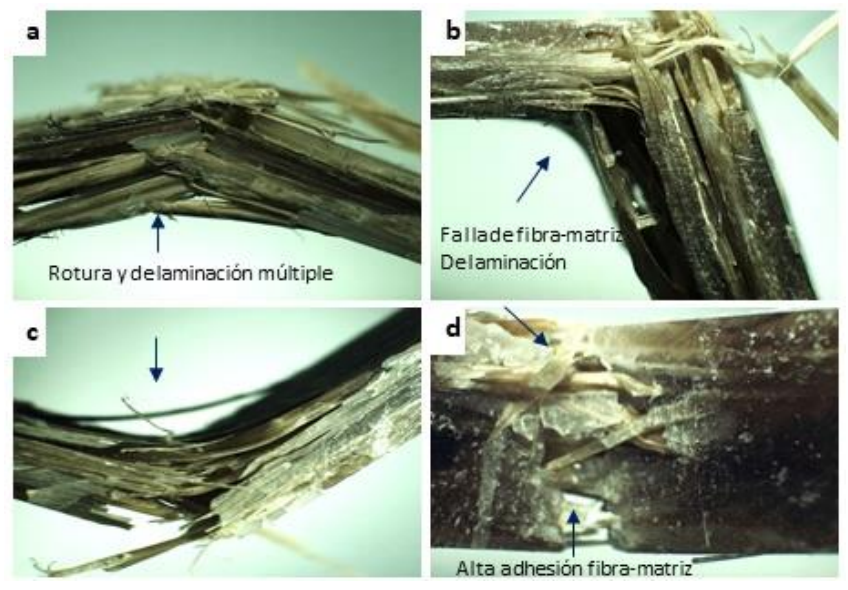

Fig.6. Fotos de la zona de fractura con un aumento de 10x de probetas de impacto con 25\% de fibra. Sin tratamiento (a). $\mathrm{MZ} 100 \mathrm{~g} / \mathrm{L} \mathrm{de} \mathrm{NaOH}$ (b) MZ 200 g/L de NaOH (c). MZ 300 g/L de $\mathrm{NaOH}$ (d).

\section{CONCLUSIONES}

En la fibra obtenida de Mauritia fexuosa se cuantificó el porcentaje de extractivos, lignina, hemicelulosa y celulosa antes y después del mercerizado donde a mayor concentración de $\mathrm{NaOH}$ se obtuvo una disminución del para extractivos, lignina y hemicelulosa respectivamente mientras el contenido de celulosa aumentó. Adicionalmente, se evidenció que a mayor concentración de $\mathrm{NaOH}$ el carácter hidrofílico de la fibra disminuye. Por otro lado, mediante FTIR, se identificó los grupos funcionales presentes en la fibra antes y después de la mercerización. Mientras, mediante microscopia electrónica de barrido (SEM), se observó los cambios topográficos de la superficie de fibras, de esta observación se determinó la disminución de impurezas, ceras y grasas además del incremento de la rugosidad de las fibras tratadas con $\mathrm{NaOH}$, en las concentraciones de $(100,200$ y 300) $\mathrm{g} / \mathrm{L}$. El incremento del porcentaje en peso de fibra Mauritia Flexuosa influyó sobre los valores de energía de impacto determinados, así los valores mayores se obtuvieron con porcentajes en peso del 25\%, siendo de estos, los compuestos con fibras no tratadas los que alcanzaron el máximo valor promedio de $39.62 \mathrm{KJ} / \mathrm{m}^{2}$. Por otro lado, para el caso de los compuestos con fibras tratadas a diferentes concentraciones de $\mathrm{NaOH}$, el incremento de este, disminuyó la energía de impacto a comparación con los compuestos con fibras no tratadas en todos los porcentajes en peso evaluados, sin embargo el mejor valor en estos casos fue a la concentración de $200 \mathrm{~g} / \mathrm{L}$ de $\mathrm{NaOH}$. Finalmente tanto el 
porcentaje en peso de fibras, como el tratamiento permitieron diferencias en los modos de daño de los compuestos ensayados; observando modos de fractura (daño matriz, tipo interlaminar y daños en fibra) de estos casos, el caso de fallo tipo interlaminar reporto estar asociado a los valores mayores de absorción de energía impacto.

\section{AGRADECIMIENTOS}

Al departamento de caracterización de materiales de la Pontificia Universidad Católica del Perú por facilitar el uso del SEM, y al laboratorio de ensayos multifuncionales, facultad de farmacia de la Universidad Nacional de Trujillo por facilitar el análisis FT-IR.

\section{REFERENCIAS}

[1] L. J. Rodríguez Sepúlveda, "Elaboración de un material biocompuesto a partir de la fibra de plátano," Universidad Nacional de ColombiaSede Manizales, 2014.

[2] M. Y. Hashim, M. N. Roslan, A. M. Amin, A. M. A. Zaidi, and S. Ariffin, "Mercerization treatment parameter effect on natural fiber reinforced polymer matrix composite: a brief review," World academy of science, engineering and technology, vol. 68, pp. 1638-1644, 2012.

[3] J. A. Taborda-Rios, L. A. Cañas-Mendoza, and J. L. TristanchoReyes, "Estudio comparativo de las propiedades mecánicas de la resina poliéster reforzada con fibra de bambú, como material sustituto de la fibra de vidrio," Dyna, vol. 84, pp. 35-41, 2017.

[4] J. M. T. González, M. A. T. Mora, and E. Santana-Castañeda, "La palma de Moriche (Mauritia flexuosa Lf;) un ecosistema estratégico," Orinoquia, vol. 15, pp. 62-70, 2011.

[5] R. da Silva Santos, A. A. de Souza, M.-A. De Paoli, and C. M. L. de Souza, "Cardanol-formaldehyde thermoset composites reinforced with buriti fibers: preparation and characterization," Composites Part A: Applied Science and Manufacturing, vol. 41, pp. 1123-1129, 2010

[6] H. L. O. Júnior, Á. G. D. O. MORAES, M. Poletto, A. J. Zattera, and S. C. Amico, "Chemical composition, tensile properties and structural characterization of buriti fiber," Cellulose Chemistry and Technology, vol. 50, pp. 15-22, 2016.

[7] K. Mylsamy and I. Rajendran, "The mechanical properties, deformation and thermomechanical properties of alkali treated and untreated Agave continuous fibre reinforced epoxy composites," Materials \& Design, vol. 32, pp. 3076-3084, 2011.

[8] L. Boopathi, P. Sampath, and K. Mylsamy, "Investigation of physical, chemical and mechanical properties of raw and alkali treated Borassus fruit fiber," Composites Part B: Engineering, vol. 43, pp. 3044-3052, 2012.

[9] K. O. Reddy, C. U. Maheswari, M. Shukla, J. Song, and A. V. Rajulu, "Tensile and structural characterization of alkali treated Borassus fruit fine fibers," Composites Part B: Engineering, vol. 44, pp. 433-438, 2013.

[10] M. F. MUÑOZ-VELEZ, M. A. HIDALGO-SALAZAR, and J. H. MINA-HERNANDEZ, "Fique fiber an alternative for reinforced plastics. Influence of surface modification," Biotecnología en el Sector Agropecuario y Agroindustrial, vol. 12, pp. 60-70, 2014.

[11] A. Balaji and K. Nagarajan, "Characterization of alkali treated and untreated new cellulosic fiber from Saharan aloe vera cactus leaves," Carbohydrate Polymers, vol. 174, pp. 200-208, 2017.

[12] A. K. Sinha, H. Narang, and S. Bhattacharya, "Effect of alkali treatment on surface morphology of abaca fibre," Materials Today: Proceedings, vol. 4, pp. 8993-8996, 2017.

[13] N. Shanmugasundaram, I. Rajendran, and T. Ramkumar, "Characterization of untreated and alkali treated new cellulosic fiber from an Areca palm leaf stalk as potential reinforcement in polymer composites," Carbohydrate Polymers, vol. 195, pp. 566-575, 2018.

[14] V. Sreenivasan, D. Ravindran, V. Manikandan, and R. Narayanasamy, "Influence of fibre treatments on mechanical properties of short
Sansevieria cylindrica/polyester composites," Materials \& Design, vol. 37, pp. 111-121, 2012.

[15] A. Athijayamani, M. Thiruchitrambalam, U. Natarajan, and B. Pazhanivel, "Effect of moisture absorption on the mechanical properties of randomly oriented natural fibers/polyester hybrid composite," Materials Science and Engineering: A, vol. 517, pp. 344353, 2009.

[16] T. Ehrman, "Standard method for determination of total solids in biomass," Chemical Analysis and Testing Task, Laboratory Analytical Procedure, vol. 242, 1994.

[17] D. Templeton and T. Ehrman, "NREL chemical analysis and testing task-laboratory analytical procedure LAP-003," Determination of Acid-Insoluble Lignin in Biomass, 1995.

[18] I. 14556, "Steel Charpy V- notch pendulum impact test-instrumented test method," ed: International Standards Organization Geneva, Switzerland, 2000 .

[19] I. ISO, "179-1: 2010, Plastics-Determination of Charpy Impact Properties, Part 1: Non-Instrumented Impact Test," International Organization for Standardization: Geneva, Switzerland, 2010.

[20] L. Y. Mwaikambo and M. P. Ansell, "Chemical modification of hemp, sisal, jute, and kapok fibers by alkalization," Journal of applied polymer science, vol. 84, pp. 2222-2234, 2002.

[21] B. C. Smith, Infrared spectral interpretation: a systematic approach: CRC press, 1998.

[22] V. S. Candido, A. C. R. da Silva, N. T. Simonassi, F. S. da Luz, and S. N. Monteiro, "Toughness of polyester matrix composites reinforced with sugarcane bagasse fibers evaluated by Charpy impact tests," Journal of Materials Research and Technology, vol. 6, pp. 334-338, 2017.

[23] H.-S. Yang, H.-J. Kim, J. Son, H.-J. Park, B.-J. Lee, and T.-S. Hwang, "Rice-husk flour filled polypropylene composites; mechanical and morphological study," Composite structures, vol. 63, pp. 305-312, 2004.

[24] M. P. Martínez, L. G. Espert, and S. Oller, "Evaluación del daño por impacto en laminados de material compuesto mediante la respuesta dinámica," Universitat Politècnica de Catalunya. Escola Tècnica Superior d'Enginyeries ..., 2012.

[25] L. J. Rodríguez, W. A. Sarache, and C. E. Orrego, "Compuestos de Poliéster Reforzados con Fibra de Plátano/Banano (Musa paradisiaca) Modificada Químicamente: Comparación con Fibra de Vidrio y Fique (Furcraea andina)," Información tecnológica, vol. 25, pp. 27-34, 2014.

[26] X. Li, L. G. Tabil, and S. Panigrahi, "Chemical treatments of natural fiber for use in natural fiber-reinforced composites: a review," Journal of Polymers and the Environment, vol. 15, pp. 25-33, 2007.

[27] P. K. Mallick, Fiber-reinforced composites: materials, manufacturing, and design: CRC press, 2007. 